\title{
Cartografía general, temática e histórica
}

\author{
Rodrigo Hidalgo y Rafael Sánchez ${ }^{2}$
}

Con este número semitemático centrado en la cartografía general, temática e histórica se ha querido rendir un homenaje al geógrafo Dr. José Ignacio González Leiva.

El profesor Leiva obtuvo en el año 1970 el título de profesor de Estado en Historia, Geografía y Educación Cívica en la Pontificia Universidad Católica de Chile. Desde sus primeros años, manifestó un fuerte interés por el área de la Geografía Matemática y la Cartografía, materias que inspirarán toda su vida académica. En 1973, gracias a una beca de la Organización de Estados Americanos realizó un post-título en Geografía Aplicada en el Centro Panamericanos de Estudios e Investigaciones Geográficas en Quito, Ecuador. A su regreso fue designado como profesor auxiliar en el naciente Instituto de Geografía de la UC. Entre 1978 y 1981, realizó sus estudios de postgrado en la Universidad de Barcelona, titulando su tesis doctoral como "La evolución de la Geografía y el desarrollo de la Cartografía temática", cuyo tutor fue el Dr. Joan Vilà i Valentí. En el año 1981 es designado profesor adjunto y en 1991 como profesor titular. Fue director del Instituto de Geografía en los periodos 1985-1991 y 1998-2006 y, decano de la Facultad de Historia, Geografía y Ciencia Política entre 1993-1999 y 2006-2013. Finalmente, dada su sobresaliente labor y experiencia académica, en el año 2013, el profesor José Ignacio Leiva es elegido para el cargo de Ombuds, cuya misión es velar y mediar por una adecuada convivencia de todas las personas que conforman la Pontificia Universidad Católica de Chile.

A lo largo de su trayectoria académica el Dr. Leiva ha participado como investigador responsable y coinvestigador en numerosos proyectos de financiamiento nacional e internacional. En todos ellos, la cartografía, como disciplina, ha sido el tema a partir del cual se ha reflexionado en torno a geografía de la población, geografía electoral, geografía urbana y organización política-administrativa del territorio. Todas estas investigaciones, cuyos resultados han quedado plasmados en numerosas publicaciones periódicas y libros, han tenido siempre una perspectiva histórica, resaltando con ello que la comprensión de los fenómenos y dinámicas territoriales responden a un contexto que se va creando y recreando. Paralelamente, su vocación por realizar una docencia significativa se ha plasmado en contribuir con instrumentos para una mejor comprensión de la ciencia geográfica y la cartografía, expresada en manuales universitarios sobre cartografía temática y geografía de Chile.

Esta destacada trayectoria académica del profesor José Ignacio González nos impulsó a reunir un conjunto de artículos que abarcaran las diferentes perspectivas del trabajo cartográfico, tanto como disciplina como instrumento auxiliar para el trabajo científico. No obstante, los otros cinco

Instituto de Geografía, Pontificia Universidad Católica de Chile (Chile). E-mail:

Instituto de Geografía, Pontificia Universidad Católica de Chile (Chile). E-mail: rsanchez@uc.cl 
artículos que se publican en este número abordan temáticas que también fueron de interés del Dr. González, tales como educación geográfica, regionalización, dinámicas urbanas y geografía general. En definitiva, este dossier demuestra el aporte que el profesor José Ignacio realizó tanto al desarrollo y consolidación del Instituto de Geografía y la Facultad de Historia, Geografía y Ciencia Política de la UC, así como también su valiosa contribución a la ciencia geográfica chilena.

El primer trabajo que inicia este semitemático es del geógrafo mexicano Dr. José' Omar Moncada Maya, quien pone su atención en la obra cartográfica realizada por los ingenieros militares en los territorios de América durante el periodo colonial. El autor considera que este corpus constituye un muestrario para entender algunos de los procesos de la ocupación territorial que tuvieron lugar a finales del siglo XVIII, pues contenían información sobre la disponibilidad de recursos naturales y humanos. A su vez, afirma que la cartografía levantada por estos ingenieros se convirtió en la base de la cartografía científica que se elaboró posteriormente por los técnicos y académicos del siglo XIX en los nacientes países iberoamericanos.

Rodrigo Moreno Jeria atrae su atención hacia la cartografía que fue publicada en 1776 en la obra "Compendio della Storia geografica, naturale e civile del Regno del Chile", todos firmados por Giovanni Fabbri, pero atribuidos a Juan Ignacio Molina. Sin embargo, el hallazgo de nuevos manuscritos demuestra que las obras fueron realizadas por otro artista, Giussepe Mancini, quien supo representar tanto el territorio de la gobernación como su capital, siguiendo las instrucciones del sacerdote chileno, es decir, puso la ciencia cartográfica al servicio de la representación e imaginario de un intelectual que recordada su patria desde el exilio.

El texto del historiador Rafael Sagredo Baeza analiza el primer mapa de Chile republicano delineado por Claudio Gay por encargo del Estado a mediados de la década de 1830. El autor enuncia que esta cartografía constituye la expresión de un proyecto institucional a través del cual los organizadores de la naciente república proyectaron su obra hacia el futuro. A su vez, este primer mapa da origen a la geografía chilena al definir el espacio nacional y diferenciarlo de otros por sus fronteras, sustrayéndolo así de la visión geográfica continental; y junto con esto, demarca el espacio donde se desenvolvería la "historia de Chile", cuyo objetivo será dotar a la naturaleza cartografiada de un pasado que la legitimara y dotara de contenido histórico.

El cuarto artículo es realizado por Alejandra Vega, quien indaga sobre un campo hasta ahora poco estudiado, las mapotecas, y para ello propone observarlas como instituciones creadas al alero de una visión particular de los mapas concebidos como espejos del mundo y discutir cómo se visibiliza ante el usuario el corpus cartográfico resguardado por estas instituciones. Para ello analiza cuatro mapotecas fundamentales existentes en Chile: la mapoteca del Archivo Nacional de Chile, la mapoteca de la Biblioteca Nacional de Chile, la mapoteca de la Facultad de Arquitectura y Urbanismo de la Universidad de Chile y la Biblioteca y Mapoteca del Instituto Geográfico Militar. La autora concluye que lo que se observa en estas mapotecas, es resultado de sucesivas inscripciones institucionales, y de las huellas de esa historia en nuestro presente.

Por último, los geógrafos argentinos Gustavo D. Buzai, Sonia L. Lanzelotti, Luis Fernando Paso Viola y Noelia Principi detallan los aspectos teórico-metodológicos que apoyaron la representación cartográfica de la alta cuenca del río Luján (Provincia de Buenos Aires, Argentina). La tarea requirió de la vinculación entre procedimientos tradicionales y las actuales tecnologías de la información geográfica (TIG) utilizadas en la construcción regional y la representación temática. 
Los autores concluyen que el estudio de la dimensión espacial de la sociedad encuentra excelentes posibilidades para analizar, comprender y poder aplicar los resultados de la investigación científica tendientes a apoyar todo proceso de toma de decisiones orientado a mejorar la relación de la sociedad con su entorno.

Tal como señalamos antes, junto con los artículos descritos, en este número semitemático se publicaron otros cinco artículos que abordan diversas temáticas y problemáticas geográficas.

María Mardones F., Mauricio Aguayo A., Ernesto Smith A. y Paulina Ruiz L. estudian las fluctuaciones de siete glaciares del Campo de Hielo Norte y su relación con variaciones climáticas decadales post Pequeña Edad del Hielo. Los autores constatan el retroceso generalizado, continuo y sincrónico en ambas laderas del Campo de Hielo. A partir de la década de 1970, las precipitaciones disminuyen y las temperaturas aumentan, y el ritmo del retroceso glacial se acelera, culminando con un colapso de gran parte de los frentes glaciares a fines del siglo pasado. Los efectos identificados es una nueva geomorfología en la zona proglacial y de zonas de peligro asociadas a procesos paraglaciales.

El artículo de Joaquín Gallastegui-Vega, Javier Vergara-Núñez, Ignacio Rojas-Rubio, Juan Gálea-Alarcón y Juan Gonzalo Saldaña-Álvarez da cuenta de los resultados de una investigación focalizada en la evaluación de los aprendizajes del campo conceptual y procedimental en el área de Geografía. Para ello se realizó una revisión de la literatura científica, el análisis curricular de los Programas de Estudio del área de la geografía y la elaboración, prueba y aplicación del test de conocimientos y habilidades fundamentales en geografía. Los autores concluyen que es imperioso pasar desde un enfoque técnico de la didáctica a un enfoque que exprese una didáctica contextual centrada en la comprensión de la experiencia del sujeto en relación con sus espacios.

El trabajo de Gisela Ariana Rausch analiza el conflicto territorial-ambiental en Santa Fe y Entre Ríos, surgido con motivo del proyecto hidroeléctrico Paraná Medio durante la década de 1990, y su resolución a favor de la coalición opositora. La autora concluye que la fuerza del discurso anti-represa se debió, en gran parte, a su conformación heterogénea, que integraba una variedad de reivindicaciones, a las cuales se fueron adhiriendo amplios sectores sociales, aunque no compartiesen los ideales del ambientalismo tradicional. A su vez, también se debió al re-escalamiento que se produjo en el modus operandi de un sector de las ONGs ambientalistas, que comenzaron a integrar redes trascendiendo la escala local, a la que, hasta ese momento, habían limitado sus luchas.

Alfredo Rehren, Arturo Orellana, Federico Arenas y Rodrigo Hidalgo realizan un ejercicio teórico sobre nuevos criterios para los procesos de regionalización en un contexto de urbanización regional o metropolización acelerada. La propuesta presenta criterios de segundo orden que podrían facilitar y respaldar la generación de nuevas regiones o la reconfiguración de otras, transitando desde una condición de simples unidades de administración y gestión hacia la constitución de verdaderas plataformas territoriales de poder, más autónomas y con mayor dotación de capital social y de conectividad.

El artículo de Tomás Errázuriz y Eduardo Valdés analiza cómo las transformaciones urbanas han afectado la experiencia de viaje de quienes diariamente se trasladan dentro de la ciudad de Talca (región del Maule, Chile) y en sus alrededores y cómo esta nueva ciudad vivida actúa sobre 
los imaginarios de los lugares que se habitan. En base al análisis de 25 entrevistas de personas que viajan regularmente por la ciudad y la confección de cartografías en donde se registran sus trayectos actuales, históricos y diversos espacios significativos, se reconoce en la mayoría de los relatos una co-existencia entre dos imaginarios de ciudad: una "Ciudad horario valle" y una "Ciudad horario punta".

Por finalizar, en este semitemático se difunden dos reseñas bibliográficas. Enrique Aliste, analiza el texto de Mauricio Onetto Pavez "Temblores de tierra en el jardín del Edén. Desastre, memoria e identidad. Chile, siglos XVI - XVIII" (2017); y Víctor Ramiro Fernández aborda el trabajo de Jamie Peck titulado "Offshore: Exploring the Worlds of Global Outsourcing" (2017). 